\title{
Multimodal assessment of right ventricle overload-metabolic and clinical consequences in pulmonary arterial hypertension
}

\author{
Remigiusz Kazimierczyk', Lukasz A. Malek², Piotr Szumowski ${ }^{3,4}$, Stephan G. Nekolla ${ }^{5}$, Piotr Blaszczak6, \\ Dorota Jurgilewicz ${ }^{4}$, Marcin Hladunski, ${ }^{3,4}$, Bozena Sobkowicz ${ }^{1}$, Janusz Mysliwiec ${ }^{4}$, Ryszard Grzywna ${ }^{5}$, \\ Wlodzimierz J. Musial ${ }^{1}$ and Karol A. Kaminski ${ }^{1,7^{*}}$ (1)
}

\begin{abstract}
Background: In pulmonary arterial hypertension (PAH) increased afterload leads to adaptive processes of the right ventricle (RV) that help to maintain arterio-ventricular coupling of RV and preserve cardiac output, but with time the adaptive mechanisms fail. In this study, we propose a multimodal approach which allows to estimate prognostic value of RV coupling parameters in PAH patients.
\end{abstract}

Methods: Twenty-seven stable PAH patients (49.5 \pm 15.5 years) and 12 controls underwent cardiovascular magnetic resonance (CMR). CMR feature tracking analysis was performed for RV global longitudinal strain assessment (RV GLS). RV-arterial coupling was evaluated by combination of RV GLS and three proposed surrogates of RV afterload — pulmonary artery systolic pressure (PASP), pulmonary vascular resistance (PVR) and pulmonary artery compliance (PAC). 18-FDG positron emission tomography (PET) analysis was used to assess RV glucose uptake presented as SUV RV/LV. Follow-up time of this study was 25 months and the clinical end-point was defined as death or clinical deterioration.

Results: Coupling parameters (RV GLS/PASP, RV GLS/PVR and RV GLS*PAC) significantly correlated with RV function and standardized uptake value $\left(S U V_{R V / L V}\right)$. Patients who experienced a clinical end-point $(n=18)$ had a significantly worse coupling parameters at the baseline visit. RV GLS/PASP had the highest area under curve in predicting a clinical end-point and patients with a value higher than $(-) 0.29 \% / \mathrm{mmHg}$ had significantly worse prognosis. It was also a statistically significant predictor of clinical end-point in multivariate analysis (adjusted $R^{2}=0.68 ; p<0.001$ ).

Conclusions: Coupling parameters are linked with RV hemodynamics and glucose metabolism in PAH. Combining CMR and hemodynamic measurements offers more comprehensive assessment of RV function required for prognostication of PAH patients.

Trial Registration: NCT03688698, 09/26/2018, retrospectively registered; Protocol ID: 2017/25/N/NZ5/02689

Keywords: Primary pulmonary hypertension, Cardiovascular magnetic resonance, Positron emission tomography, Prognosis, Coupling

\footnotetext{
*Correspondence: fizklin@wp.pl

${ }^{7}$ Department of Population Medicine and Lifestyle Diseases Prevention,

Medical University of Bialystok, Waszyngtona 13a, Białystok 15-269, Poland

Full list of author information is available at the end of the article
}

\begin{abstract}
Introduction
Pulmonary arterial hypertension (PAH) is a progressive disease, in which declining right ventricular (RV) function with accompanying uncoupling to the pulmonary circulation is a turning point of clinical worsening. Therefore, accurate assessment of RV systolic
\end{abstract} permits use, sharing, adaptation, distribution and reproduction in any medium or format, as long as you give appropriate credit to the original author(s) and the source, provide a link to the Creative Commons licence, and indicate if changes were made. The images or other third party material in this article are included in the article's Creative Commons licence, unless indicated otherwise in a credit line to the material. If material is not included in the article's Creative Commons licence and your intended use is not permitted by statutory regulation or exceeds the permitted use, you will need to obtain permission directly from the copyright holder. To view a copy of this licence, visit http://creativecommons.org/licenses/by/4.0/. The Creative Commons Public Domain Dedication waiver (http://creativeco mmons.org/publicdomain/zero/1.0/) applies to the data made available in this article, unless otherwise stated in a credit line to the data. 
function, especially before significant clinical deterioration is crucial for PAH patients [1-3]. The RV adapts to the increasing vascular load by enhancing contractility according to Frank-Starling law, preserving RV-arterial coupling and maintaining blood flow to peripheral demand $[4,5]$. The concept of coupling mainly refers to the relationship between ventricular contractility and afterload thus alterations of this phenomenon may be an early marker of RV failure [6]. The gold standard to assess $\mathrm{RV}$-arterial coupling requires invasive procedure to obtain pressure-volume loop-derived end-systolic elastance (Ees) to arterial elastances (Ea) ratio. This ratio (Ees/Ea) gives direct quantification of $\mathrm{RV}$-arterial coupling [4]. However, measuring these parameters via pressure-volume loops is technically demanding and cannot be performed routinely during regular PAH patients' assessments.

Cardiovascular magnetic resonance (CMR) imaging is the gold standard, noninvasive method for RV functional assessment. RV contractility may be described by various CMR parameters like RV ejection fraction (RVEF), tricuspid annular plane systolic excursion (TAPSE), fractional area change (FAC) or myocardial strain [7-9]. RV global longitudinal strain (GLS) obtained from CMR feature tracking was recently associated with worse outcomes in PAH [10-12]. RV GLS seems to be less load independent than RVEF. It also correlates with diastolic stiffness, and thus could reflect better RV contractility in overloaded RV in PAH [13, 14].

The RV afterload is composed of static and pulsatile components. According to Windkessel model, pulmonary arterial resistance (PVR) or pulmonary artery pressures represent only the mean resistive or static afterload (an opposition to forward flow) when the pulsatile component may be represented by pulmonary artery compliance parameter-PAC [15].

Combining RV contractility with estimations of its afterload, especially using multimodal techniques, seems to be a novel approach allowing not only to better understand RV hemodynamics but also creates possibilities of new prognostic markers.

In $\mathrm{PAH}$, the RV also undergoes a number of metabolic changes, including the shift to the less energetically efficient process of glycolysis [3]. This results in a compensatory upregulation of glucose flux into RV myocytes. The flux can be quantified in positron emission tomography (PET) imaging and presented as ${ }^{18} \mathrm{~F}$-fluorodeoxyglucose (FDG) standardized uptake value (SUV) parameter [16]. In severe PAH patients, due to higher pressure overload of RV maladaptive increase of the FDG uptake is observed [17]. Recently, we confirmed that FDG uptake (presented as $\mathrm{SUV}_{\mathrm{RV} / \mathrm{LV}}$ ) correlates with $\mathrm{PAH}$ progression and unfavourable outcomes [18].
In this research, we chose CMR-derived RV GLS as the main parameter of RV contractility. As surrogates of a RV afterload, we used systolic pulmonary artery pressure (PASP); PVR and PAC, all obtained from right heart catheterization (RHC) and CMR.

The aim of this study was to evaluate whether relating RV shortening, as assessed by feature tracking CMR, to the force developed, as expressed by PASP/PVR/PAC, could translate into a more accurate estimation of RV performance status and, consequently, in an improvement in risk prediction accuracy. We also hypothesized that presented above coupling parameters may be related to alterations in RV glucose uptake both preceding clinical deterioration.

\section{Methods}

\section{Population characteristics}

We enrolled 27 clinically stable, adult patients diagnosed with PAH in World Health Organization (WHO) class II or III. The diagnosis of pre-capillary pulmonary hypertension $(\mathrm{PH})$ was confirmed by RHC [mean pulmonary artery pressure $(\mathrm{mPAP}) \geq 25 \mathrm{mmHg}$, pulmonary artery wedge pressure (PAWP) $\leq 15 \mathrm{mmHg}$ ] and the use of an algorithm that included a perfusion lung scan, echocardiography, respiratory function tests, and computer tomography to exclude secondary $\mathrm{PH}$ causes according to European guidelines [1]. The exclusion criteria were following: patients in grade IV WHO class, Eisenmenger physiology, PAH associated with prevalent systemicto-pulmonary shunts due to moderate to large defects (according to European guidelines) [1], group II, III, IV, $\mathrm{V}$ of $\mathrm{PH}$ and contraindications to CMR. The control group consisted of 12 healthy subjects who were selected based on sex and age. During the baseline evaluation, we performed a physical examination, six-minute walk test (6MWT), laboratory tests e.g. serum B-type natriuretic peptide (BNP), blood count and renal function parameters.

RHC was carried out only in the PAH group with a standard technique within median 4 [2-6] days of PET/ CMR scans using a balloon-tipped 7F pulmonary artery catheter to assess systolic pulmonary artery pressure (sPAP), diastolic pulmonary artery pressure (dPAP), mPAP and PAWP. Cardiac output (CO) was measured by thermodilution method. PVR was calculated with the formula $\mathrm{PVR}=(\mathrm{mPAP}-\mathrm{PAWP}) /(\mathrm{CO})$ and expressed in Wood's units (WU) and PAC parameter with formula $\mathrm{PAC}=$ stroke volume $(\mathrm{SV}) /(\mathrm{sPAP}-\mathrm{dPAP}) . \mathrm{RV}$-arterial coupling was evaluated by the ratio of RV GLS/PASP; RV GLS/PVR; RV GLS*PAC.

Clinical follow-up was 25 months. Death, WHO class worsening, hospitalisation due to $\mathrm{PH}$ or right heart failure as assessed by European Society of Cardiology (ESC) 
criteria were used as composite clinical endpoint (CEP) for Kaplan-Meier analysis. All enrolled patients were rehospitalized only in our Department and their clinical state or death were evaluated/confirmed by PAH specialists (co-authors of this paper). The study was approved by the local Bioethics Committee at Medical University of Bialystok (R-I-002/140/2017). All patients gave written informed consent for participating in the study, including taking and storage of blood samples. The investigation conforms with the principles outlined in the Declaration of Helsinki" (Br Med J 1964; ii: 177).

\section{PET/CMR imaging}

Simultaneous PET/CMR imaging was performed with a 3T Biograph mMR hybrid system (Siemens, Healthineers Erlangen, Germany) at the baseline visit. CMR studies were analysed using a dedicated workstation and software (Horos v 3.3.5 with validated MRHeart plugin) [19]. CMR results were not known to treating physician and did not affect the decision about the therapy, simultaneously physician analyzing the CMR was not aware of the patient's clinical state.

Systolic function assessment was based on cine balanced steady state free precession (bSSFP) short axis images from the tricuspid valve insertion point to the apex to encompass the entire RV. The imaging parameters were the following: field of view $360 \mathrm{~mm}$, matrix $256 \times 256$, repetition time approximately $40.7 \mathrm{~ms}$, echo time $1.49 \mathrm{~ms}$, flip angle 50 degrees, slice thickness $6 \mathrm{~mm}$, gap $1.2 \mathrm{~mm}$, in-plane image resolution $1.4 \times 1.4 \times 6 \mathrm{~mm}^{3}$, temporal resolution 25 phases per cardiac cycle.

Short axis bSSFP cine images were initially previewed from the base to the apex in a cinematic mode; then endocardial and epicardial contours for RV end-diastole and end-systole were manually traced. Trabeculae were considered as ventricle cavities. The delineated contours were used for the quantification of RV variables, which were then indexed to body surface area (BSA). RV FAC was obtained from formula (end-diastolic area - end-systolic area)/end-diastolic area $\times 100$.

RV strain analysis was performed with the use of a dedicated software (QStrain, Mass Medis, Leiden, The Netherlands). For the purpose of analysis endocardial borders of the RV were manually traced in end-systole and enddiastole on 4-chamber view and used for generation of RV GLS [14].

Heart glucose metabolism was assessed with FDG as a tracer in PET. One hour after $i v$ injection of FDG (185 MBq $\pm 15 \mathrm{MBq})$, myocardial PET imaging was performed as previously described [18]. Its uptake was quantified as mean SUV for both the left ventricle (LV) and $\mathrm{RV}$ and presented as a ratio of $\mathrm{SUV} \mathrm{RV}_{\mathrm{LV}}$.

\section{Statistical analysis}

The distribution of the variables was checked using the Kolmogorov-Smirnov test. The data are expressed as a mean \pm standard deviation (SD) or median [interquartile range] as appropriate. Statistical analysis was performed using Student's $t$-test or Mann-Whitney U test for continuous data depending on distribution. Spearman's correlation coefficient was used to examine the relationship between two continuous variables. Benjamini-Hochberg correction was used to account for multiple comparisons in correlation analysis. Univariable and multivariable Cox proportional hazards regression analyses were performed to identify independent variables associated with end-point. Receiver operator characteristic curves (ROC) were plotted to determine the area under the curve (AUC) and sensitivity and specificity of the optimal cut-offs (binomial method). DeLong's test was used to compare two AUC results. To investigate the occurrence of clinical endpoints Kaplan Meier method with logrank test was implemented. $\mathrm{p}<0.05$ was deemed statistically significant. A statistical software package STATA13 (Stata Corporation, College Station, Texas, USA) was used for the analysis.

\section{Results}

\section{Patients' characteristics}

Twenty-seven stable PAH patients $49.5 \pm 15.5$ years (idiopathic/heritable $n=18$; connective tissue diseases $n=4$; associated with congenital small/coincidental defects $\mathrm{n}=5)$ and 12 healthy subjects (control group) selected based on age and sex ( $44.8 \pm 13.5$ years, 8 females) were enrolled in the study. Five PAH patients (18\%) were incident cases while the rest of the study group were prevalent-receiving PAH specific treatment at the time of survey. Most of them were in the WHO functional Class III $(62 \%, \mathrm{n}=17)$ and 10 patients $(38 \%)$ were in WHO functional Class II; majority of patients were women $(62 \%, n=17)$. According to 1-year mortality risk groups presented in ESC guidelines [7], nineteen patients (70\%) were at intermediate risk; five patients (19\%) at low risk and three patients $(11 \%)$ at high risk. The mean 6-min walk test distance was $387 \pm 103 \mathrm{~m}$ and median plasma BNP level was $269 \mathrm{pg} / \mathrm{ml}$. Groups characteristics' and PET/CMR and RHC results are presented in Table 1.

\section{CMR parameters and RV function}

In the PAH group, RV dimensions and mass were significantly higher than in the healthy control group and RVEF was significantly lower (Table 1 ). LV ejection fraction (LVEF) did not differ between both groups. RV function presented as RV GLS was significantly less negative (worse) in PAH group than in the healthy control group 
Table 1 Basic characteristics of pulmonary arterial hypertension (PAH) group and healthy controls

\begin{tabular}{|c|c|c|}
\hline & $\begin{array}{l}\text { Pulmonary artery } \\
\text { hypertension }\end{array}$ & $\begin{array}{l}\text { Healthy } \\
\text { controls }\end{array}$ \\
\hline Subjects, $\mathrm{n}$ & 27 & 12 \\
\hline Age, years & $49.5 \pm 15.5$ & $44.7 \pm 13.5$ \\
\hline Sex (females), \% (n) & $62(17)$ & $67(8)$ \\
\hline $\mathrm{BSA}, \mathrm{m}^{2}$ & $1.8 \pm 0.2$ & $1.8 \pm 0.2$ \\
\hline 6MWT, m & $387 \pm 103$ & \\
\hline $\mathrm{BNP}, \mathrm{pg} / \mathrm{ml}$ & $269[22-925]$ & \\
\hline \multicolumn{3}{|l|}{ Aetiology } \\
\hline IPAH/HPAH, \% (n) & $66(18)$ & \\
\hline CTDPH, \% (n) & $14(4)$ & \\
\hline $\mathrm{CHDPH}, \%(\mathrm{n})$ & $20(5)$ & \\
\hline \multicolumn{3}{|l|}{ Therapy } \\
\hline PDE5 inhibitors, \% (n) & $40(11)$ & \\
\hline ERA, \% (n) & $11(3)$ & \\
\hline Prostacyclins, \% (n) & $20(5)$ & \\
\hline Dual PDE5 inhibitor + ERA, \% (n) & $29(8)$ & \\
\hline \multicolumn{3}{|l|}{ Hemodynamics } \\
\hline sPAP, mmHg & $77.4 \pm 27.7$ & \\
\hline $\mathrm{dPAP}, \mathrm{mmHg}$ & $32.3 \pm 14.1$ & \\
\hline mPAP, mmHg & $48.6 \pm 17.6$ & \\
\hline PCWP, mmHg & $10.3 \pm 2.4$ & \\
\hline $\mathrm{DPG}, \mathrm{mmHg}$ & $22.9 \pm 13.5$ & \\
\hline PVR, Wood Units & $8.7 \pm 5.4$ & \\
\hline $\mathrm{Cl}, \mathrm{L} / \mathrm{min} / \mathrm{m}^{2}$ & $2.7 \pm 0.8$ & \\
\hline $\mathrm{RAP}, \mathrm{mmHg}$ & $8.6 \pm 3.5$ & \\
\hline \multicolumn{3}{|l|}{ RV functional parameters (CMR) } \\
\hline RVEF, \% & $44.8 \pm 10.2^{\wedge}$ & $63.8 \pm 5.8$ \\
\hline TAPSE, $\mathrm{mm}$ & $19 \pm 4.3^{\wedge}$ & $24.9 \pm 2.4$ \\
\hline $\mathrm{RV} E D V / B S A, \mathrm{ml} / \mathrm{m}^{2}$ & $117.8 \pm 29.9 \wedge$ & $73.6 \pm 12.2$ \\
\hline RVESV/BSA, $\mathrm{ml} / \mathrm{m}^{2}$ & $66.5 \pm 27.1 \wedge$ & $28.2 \pm 9.6$ \\
\hline $\mathrm{RV}$ mass/BSA, $\mathrm{g} / \mathrm{m}^{2}$ & $42.9 \pm 17.1 \wedge$ & $23.8 \pm 4.9$ \\
\hline $\mathrm{PAC}, \mathrm{ml} / \mathrm{mmHg}$ & $2.5 \pm 1.6$ & \\
\hline GLS, \% & $-16.4 \pm 7.4 \wedge$ & $-30.7 \pm 9.7$ \\
\hline \multicolumn{3}{|l|}{ Myocardial metabolism (PET) } \\
\hline $\mathrm{SUV}_{\mathrm{RV}} / \mathrm{SUV} \mathrm{LV}_{\mathrm{LV}}$ ratio & $1.03 \pm 0.76 \wedge$ & $0.19 \pm 0.08$ \\
\hline
\end{tabular}

Data are presented as mean \pm standard deviation or median [interquartile range]

6MWD 6-minute walk test distance, $B S A$ body surface area, $B N P$ brain natriuretic peptide, $\mathrm{Cl}$ cardiac index, $C H D P A H$ congenital heart disease related pulmonary arterial hypertension, CMR cardiovascular magnetic resonance, CTDPAH connective tissue disease related pulmonary arterial hypertension, DPG diastolic pulmonary gradient, $A P A P$ diastolic pulmonary artery pressure, $E D V$ enddiastolic volume, ESV end-systolic volume, ERA endothelin receptor antagonist, GLS global longitudinal strain, HPAH heritable pulmonary arterial hypertension, IPAH idiopathic pulmonary arterial hypertension, GLS global longitudinal strain, mPAP mean pulmonary artery pressure, $P A C$ pulmonary arterial compliance, $P A H$ pulmonary arterial hypertension, $P A S P$ pulmonary artery systolic pressure, $P C W P$ pulmonary capillary wedge pressure, $P D E 5$ phosphodiesterase type 5, PET positron emission tomography, PVR pulmonary vascular resistance, SPAP systolic pulmonary artery pressure, $R A P$ right atrial pressure, $R V$ right ventricle, $R V E F$ right ventricle ejection fraction, $S U V$ standardized uptake value, $\mathrm{SVO}_{2}$ mixed venous oxygen saturation, TAPSE cardiac magnetic resonance tricuspid annular plane systolic excursion, WHO World Health Organisation

$\wedge$ Statistically significant difference between $\mathrm{PAH}$ and control groups, $\mathrm{p}<0.005$
$(-16.4 \pm 7.4 \%$ vs $-30.7 \pm 9.7 \%, \mathrm{p}<0.001)$. In the study group, RV GLS correlated with hemodynamic parameters from RHC - mPAP $(\mathrm{r}=0.37, \mathrm{p}=0.05)$ and PVR $(\mathrm{r}=0.53, \mathrm{p}=0.003)$, Fig. 1 ; from CMR - with TAPSE $(\mathrm{r}=-0.54, \mathrm{p}=0.01)$ and $\operatorname{RVEF}(\mathrm{r}=-0.7, \mathrm{p}<0.001)$.

Mean values of the RV-pulmonary artery coupling parameters in PAH group were-RV GLS/PASP $(-) 0.25 \pm 0.17 \% / \mathrm{mmHg} ; \mathrm{RV}$ GLS/PVR $-(-) 3.3 \pm 2.8 \% /$ WU and RV GLS*PAC - (-)42.9 $\pm 32.1 \% * \mathrm{mmHg} / \mathrm{ml}$.

All three parameters significantly correlated with established CMR-derived RV systolic function variables-RVEF and FAC (Table 2). Interestingly, three coupling parameters correlated also with BNP levels but not with functional PAH prognosis predictors-WHO class and $6 \mathrm{MWT}$.

\section{RV_coupling and RV metabolism}

In PET analysis, the cardiac FDG uptake parameter $\mathrm{SUV}_{\mathrm{RV} / \mathrm{LV}}$ was significantly higher in PAH group than in healthy controls $(1.03 \pm 0.76$ vs $0.19 \pm 0.08, \mathrm{p}<0.001)$. It also correlated with parameters of RV dysfunction e.g. RVEF $(r=-0.52, p<0.001)$ and mPAP $(r=0.77$, $\mathrm{p}<0.001)$.

All three coupling parameters (RV GLS/PASP, RV GLS/ PVR and RV GLS*PAC) significantly correlated with $\mathrm{SUV}_{\mathrm{RV} / \mathrm{LV}}$, Table 2, Fig. 2. Importantly, no significant correlation between non-indexed RV GLS and $S U V_{R V / L V}$ was found $(\mathrm{r}=0.36, \mathrm{p}=0.11)$.

In univariate logistic regression analysis, RV GLS, RV GLS/PASP, WHO class, 6MWT distance, CVP were associated with $\mathrm{SUV}_{\mathrm{RV} / \mathrm{LV}}$ dichotomized at 1 (higher glucose uptake of RV than of LV), which value has also significant prognostic value $[16,18]$.

\section{Survival analysis}

After 25 months, 18 patients (66\%) met a composite endpoint. Five patients died, 13 patients had WHO class worsening (ten patients from WHO class II to III and three patients from WHO class III to IV) including four patients who required initiation of parenteral prostacyclin analogue. Mean time to clinical worsening was $16.9 \pm 8.0$ months.

Patients who reached CEP had a significantly higher RV GLS and all three coupling parameters at the baseline visit than patients who remained stable (Table 3). There were also significant differences in CMR and RHCderived parameters but not in 6MWT distance or BNP levels. In comparison to coupling parameters difference in non-indexed RV afterload surrogate-PAC was not statistically significant.

ROC analysis revealed highest area under the curve (AUC) in predicting CEP for RV GLS/PASP ratio, Table 4. Importantly, AUC for coupled parameter RV 

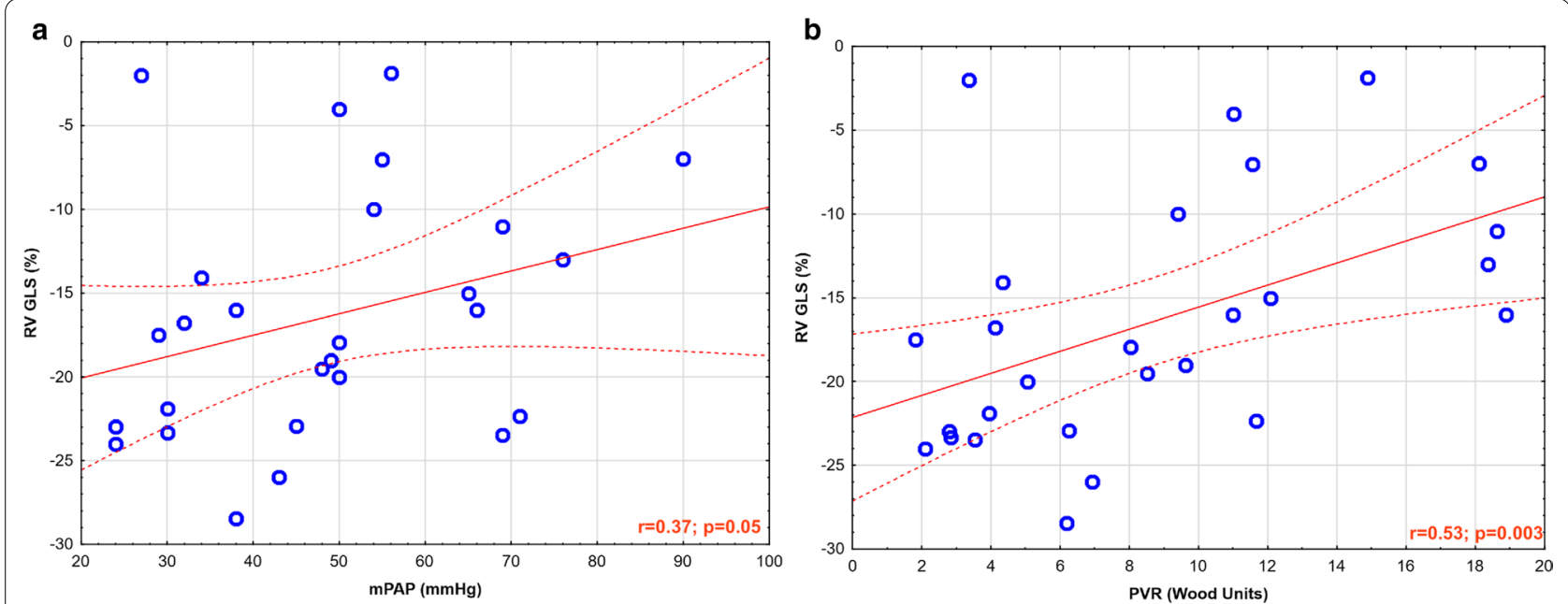

Fig. 1 Spearman correlations between right ventricular (RV) global longitudinal strain (GLS) (from cardiovascular magnetic resonance (CMR)) and a mean pulmonary artery pressure (MPAP) and $\mathbf{b}$ pulmonary vascular resistance (PVR), both obtained from right heart catheterization (RHC) in the pulmonary artery hypertension (PAH) group. CMR cardiac magnetic resonance; GLS global longitudinal strain; mPAP mean pulmonary arterial hypertension; $P A H$ pulmonary arterial hypertension; $P V R$ pulmonary vascular resistance, $R H C$ right heart catheterization; $R V$ right ventricle.

Table 2 Spearman correlations between RV-arterial coupling parameters and other parameters of RV function and metabolism

\begin{tabular}{|c|c|c|c|}
\hline & RV GLS/PASP & RV GLS/PVR & RV GLS*PAC \\
\hline WHO class & $r=0.28 ; p=0.15$ & $r=0.28 ; p=0.14$ & $r=0.26 ; p=0.19$ \\
\hline 6MWT distance & $r=-0.22 ; p=0.25$ & $r=-0.29 ; p=0.13$ & $r=-0.22 ; p=0.24$ \\
\hline BNP level & $r=0.31 ; p=0.10$ & $r=0.34 ; p=0.07$ & $r=0.30 ; p=0.12$ \\
\hline TAPSE (CMR) & $r=-0.15 ; p=0.43$ & $r=-0.15 ; p=0.42$ & $r=-0.08 ; p=0.65$ \\
\hline RVEF (CMR) & $r=-0.75 ; p=0.000005^{\wedge}$ & $r=-0.71 ; p=0.00002^{\wedge}$ & $r=-0.73 ; p=0.00001 \wedge$ \\
\hline FAC (CMR) & $r=-0.78 ; p=0.000002^{\wedge}$ & $r=-0.77 ; p=0.000001 \wedge$ & $r=-0.69 ; p=0.00004 \wedge$ \\
\hline RV mass/BSA (CMR) & $r=0.59 ; p=0.001 \wedge$ & $r=0.60 ; p=0.0008^{\wedge}$ & $r=0.47 ; p=0.012^{\wedge}$ \\
\hline $\mathrm{Cl}(\mathrm{RHC})$ & $r=-0.54 ; p=0.003 \wedge$ & $r=-0.63 ; p=0.0004 \wedge$ & $r=-0.48 ; p=0.01 \wedge$ \\
\hline$S_{S U V}$ RV/LV $(P E T)$ & $r=0.67 ; p=0.0002^{\wedge}$ & $r=0.55 ; p=0.002^{\wedge}$ & $r=0.69 ; p=0.00006 \wedge$ \\
\hline
\end{tabular}

6MWT six minute walk test distance, BNP serum brain natriuretic peptide, $B S A$ body surface area, $C l$ cardiac index, $C M R$ cardiac magnetic resonance, GLS global longitudinal strain, FAC fractional area change, $L V$ left ventricle, $P A C$ pulmonary arterial compliance, PASP pulmonary artery systolic pressure, $P E T$ positron emission tomography, $P V R$ pulmonary vascular resistance, $R V$ right ventricle, $R V E F$ right ventricle ejection fraction, SUV standardized uptake value, TAPSE tricuspid annular plane systolic excursion, WHO World Health Organisation

$\wedge$ p-value significant (lower than 0.05) after Benjamini-Hochberg correction

GLS/PASP was numerically higher than for RV GLS alone $(0.96$ [0.88-1] vs 0.87 [0.73-1], $\mathrm{p}=\mathrm{ns})$. Patients with RV GLS/PASP cut-off value (ROC analysis) higher than $(-) 0.29 \% / \mathrm{mmHg}$ had worse prognosis, log-rank test, $\mathrm{p}=0.0008$, Fig. 3 .

Furthermore, ROC analysis showed that also RV GLS*PAC has a comparable predictive value for the composite endpoint as RV GLS/PVR $(\mathrm{p}=0.33)$ and numerically higher than PAC alone (0.89 vs 0.72 , $\mathrm{p}=0.18$ ). Patients with an RV GLS*PAC value higher than $(-) 48.35 \% * \mathrm{mmHg} / \mathrm{ml}$ had significantly worse prognosis in Kaplan-Meier analysis, log-rank test, $\mathrm{p}=0.004$.
In univariable logistic regression analysis all three coupling parameters were significantly associated with CEP. However, multivariable stepwise regression analysis demonstrated that in the model predicting time to clinical end-point RV GLS/PASP remained statistically significant (Table 5). The model containing RV GLS/PASP beside other PAH prognostic parameters (WHO class, RVEF, mPAP, SUV $V_{\text {RV/LV }}$ ) had higher adjusted $R^{2}$ than model with sole RV GLS $\left(\mathrm{R}^{2}=0.68\right.$ vs 0.62$)$.

We also performed similar sub analysis for RVEF as a surrogate of RV contractility. RVEF was significantly lower in CEP patients( $40.9 \pm 8.8$ vs $52.5 \pm 8.4, \mathrm{p}=0.004)$ and there were statistically significant differences in 


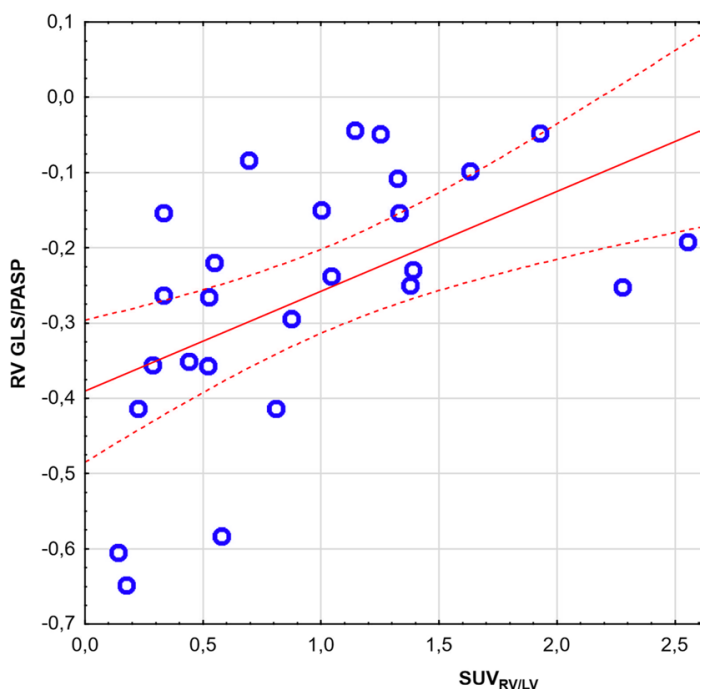

Fig. 2 Spearman correlation between RV GLS/PASP and SUV RV/LV PAH group. GLS global longitudinal strain; LV left ventricle; PAH pulmonary arterial hypertension; PASP pulmonary artery systolic pressure; RV right ventricle; SUV standardized uptake value.
Table 4 Comparison of area under curve (AUC) of various parameters for prediction of composite end-point (ROC analysis)

\begin{tabular}{lll}
\hline Value & $\begin{array}{l}\text { AUC (95\% confidence } \\
\text { interval) }\end{array}$ & p-value \\
& $0.96(0.88-1)$ & $\mathrm{p}<0.001$ \\
\hline RV GLS/PASP & $0.94(0.86-1)$ & $\mathrm{p}<0.001$ \\
RV GLS/PVR & $0.89(0.76-1)$ & $\mathrm{p}<0.001$ \\
RV GLS*PAC & $0.87(0.73-1)$ & $\mathrm{p}<0.001$ \\
RV GLS & $0.84(0.67-1)$ & $\mathrm{p}<0.001$ \\
SUV RV/LV & $0.83(0.65-1)$ & $\mathrm{p}<0.001$ \\
RVEF & $0.88(0.75-1)$ & $\mathrm{p}=0.04$ \\
PVR & $0.72(0.52-0.92)$ & $\mathrm{p}=0.03$ \\
PAC & $0.79(0.62-0.96)$ & $\mathrm{p}=0.005$ \\
TAPSE/RVSP (echo/echo) & $0.76(0.58-0.94)$ & $\mathrm{p}=0.004$ \\
TAPSE/RVSP (CMR/echo) & $0.89(0.77-1)$ & $\mathrm{p}<0.001$ \\
RV GLS/RVSP (CMR/echo) & $0.78(0.58-0.96)$ & $\mathrm{p}=0.002$ \\
RVEF/RVSP (CMR/echo) &
\end{tabular}

CMR cardiac magnetic resonance, echo echocardiography, GLS global longitudinal strain, $L V$ left ventricle, $P A C$ pulmonary arterial compliance, $P A S P$ pulmonary artery systolic pressure, $P E T$ positron emission tomography, $P V R$ pulmonary vascular resistance, $R V$ right ventricle, $R V E F$ right ventricle ejection fraction, RVSP right ventricle systolic pressure obtained by echocardiography, SUV standardized uptake value, TAPSE tricuspid annular plane systolic excursion

Table 3 Comparison between patients with composite endpoint (CEP+) and without (CEP-)

\begin{tabular}{|c|c|c|c|}
\hline & $\begin{array}{l}\text { CEP }(+) \\
\text { patients }\end{array}$ & $\begin{array}{l}\text { CEP (-) } \\
\text { patients }\end{array}$ & p-value \\
\hline Patients, $\mathrm{n}$ & 18 & 9 & \\
\hline BNP, pg/ml & 241 [22-925] & $62[36-435]$ & 0.19 \\
\hline 6MWT distance, $\mathrm{m}$ & $358.8 \pm 97.2$ & $443.6 \pm 95.4$ & 0.06 \\
\hline $\mathrm{SUV}_{\mathrm{RV}} / \mathrm{SUV}$ LV ratio & $1.29 \pm 0.78$ & $0.51 \pm 0.39$ & 0.003 \\
\hline RVEF, \% & $40.9 \pm 8.8$ & $52.5 \pm 8.4$ & 0.004 \\
\hline TAPSE, mm & $17.9 \pm 4.0$ & $21.3 \pm 4.2$ & 0.07 \\
\hline $\mathrm{PAC}, \mathrm{ml} / \mathrm{mmHg}$ & $2.0 \pm 0.9$ & $3.3 \pm 2.3$ & 0.06 \\
\hline mPAP, mmHg & $56.3 \pm 15.8$ & $33.1 \pm 8.2$ & $<0.001$ \\
\hline PVR, wood units & $10.9 \pm 5.3$ & $4.3 \pm 2.3$ & $<0.001$ \\
\hline RV GLS, \% & $-13.5 \pm 7$ & $-22.2 \pm 3.8$ & 0.001 \\
\hline RV GLS/PASP, \%/mmHg & $-0.16 \pm 0.08$ & $-0.44 \pm 0.11$ & $<0.001$ \\
\hline RV GLS/PVR, \%/WU & $-1.75 \pm 1.68$ & $-6.4 \pm 3.1$ & $<0.001$ \\
\hline RVGLS*PAC, \%*mmHg/ml & $-26 \pm 16.7$ & $-75.1 \pm 54.3$ & $<0.001$ \\
\hline RVEF/PASP, \%*mmHg & $0.51 \pm 0.21$ & $1.02 \pm 0.36$ & $<0.001$ \\
\hline RVEF/PVR, \%*WU & $5.37 \pm 4.16$ & $15.72 \pm 8.26$ & $<0.001$ \\
\hline RVEF*PAC, \%/mmHg/ml & $85.3 \pm 50.4$ & $181.9 \pm 133.1$ & 0.006 \\
\hline TAPSE/RVSP (echo/echo) & $0.30 \pm 0.17$ & $0.44 \pm 0.21$ & 0.012 \\
\hline TAPSE/RVSP (CMR/echo) & $0.28 \pm 0.16$ & $0.55 \pm 0.21$ & 0.03 \\
\hline RV GLS/RVSP (CMR/echo) & $-0.22 \pm 0.19$ & $-0.57 \pm 0.21$ & 0.03 \\
\hline RVEF/RVSP (CMR/echo) & $0.68 \pm 0.39$ & $1.26 \pm 0.58$ & 0.01 \\
\hline
\end{tabular}

6MWT six minute walk test distance, $B N P$ serum brain natriuretic peptide, $B S A$ body surface area, CEP composite end-point, $C l$ cardiac index, CMR cardiac magnetic resonance, echo echocardiography, GLS global longitudinal strain, $F A C$ fractional area change, $L V$ left ventricle, $M P A P$ mean pulmonary artery pressure, $P A C$ pulmonary arterial compliance, $P A S P$ pulmonary artery systolic pressure, $P E T$ positron emission tomography, $P V R$ pulmonary vascular resistance, $R V$ right ventricle, $R V E F$ right ventricle ejection fraction, $R V S P$ right ventricle systolic pressure obtained by echocardiography, SUV standardized uptake value, TAPSE tricuspid annular plane systolic excursion, WHO World Health Organisation

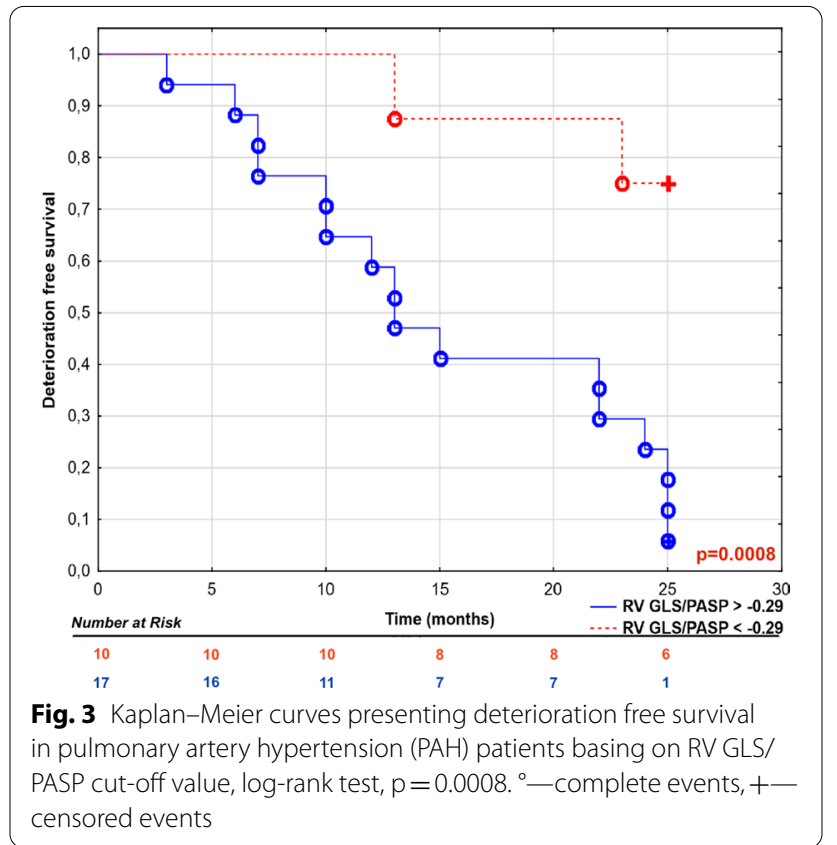

coupling parameters-RVEF/PASP; RVEF/PVR and RVEF*PAC between both groups, Table 3. Also, RVEF was a significant parameter in univariable regression and ROC analysis.

\section{Non-invasive coupling approach}

Additional analysis of fully non-invasive coupling approach was also conducted, where TAPSE (derived from echo 
Table 5 Univariable and multivariable logistic regression analysis for the composite endpoint

\begin{tabular}{|c|c|c|c|}
\hline \multirow[t]{2}{*}{ Value } & \multicolumn{3}{|c|}{ Univariable analysis } \\
\hline & HR & $95 \% \mathrm{Cl}$ & $\mathrm{p}$-value \\
\hline WHO Class & 12.34 & $1.53-95.49$ & 0.01 \\
\hline Age & 0.98 & $0.93-1.03$ & 0.50 \\
\hline BSA & 0.03 & $0.01-2.88$ & 0.13 \\
\hline BNP & 1.03 & $0.99-1.08$ & 0.13 \\
\hline 6MWT & 0.99 & $0.97-1$ & 0.05 \\
\hline \multicolumn{4}{|l|}{ PET parameters } \\
\hline$S U V_{R V} / S U V_{L V}$ ratio & 17.84 & $1.48-214.8$ & 0.02 \\
\hline \multicolumn{4}{|l|}{ CMR parameters } \\
\hline RVEF & 0.83 & $0.71-0.96$ & 0.01 \\
\hline RV mass/BSA & 1.09 & $1.06-1.18$ & 0.05 \\
\hline PAC & 0.50 & $0.23-1.21$ & 0.09 \\
\hline \multicolumn{4}{|l|}{ RHC parameters } \\
\hline mPAP & 1.67 & $1.04-1.30$ & 0.008 \\
\hline PCWP & 1.04 & $0.74-1.47$ & 0.79 \\
\hline DPG & 1.31 & $0.99-1.44$ & 0.05 \\
\hline RAP & 1.61 & $1.03-2.51$ & 0.03 \\
\hline $\mathrm{Cl}$ & 0.24 & $0.05-1.07$ & 0.06 \\
\hline PVR & 1.56 & $1.08-2.25$ & 0.01 \\
\hline $\mathrm{SvO}_{2}$ & 0.99 & $0.87-1.21$ & 0.89 \\
\hline RV GLS & 1.38 & $1.05-1.81$ & 0.01 \\
\hline RV GLS/PASP* & 19.88 & $3.59-1099$ & 0.003 \\
\hline RV GLS/PVR & 2.31 & $1.20-4.45$ & 0.01 \\
\hline RV GLS*PAC & 1.09 & $1.01-1.18$ & 0.01 \\
\hline
\end{tabular}

6MWD 6-minute walking test distance, BSA body surface area, BNP brain natriuretic peptide, $\mathrm{Cl}$ cardiac index, DPG diastolic pulmonary gradient, GLS global longitudinal strain, $M P A P$ mean pulmonary artery pressure, $P A C$ pulmonary arterial compliance, $P A S P$ pulmonary artery systolic pressure, $P C W P$ pulmonary capillary wedge pressure, $P E T$ positron emission tomography, $P V R$ pulmonary vascular resistance, $R A P$ right atrial pressure, $R V$ right ventricle, $R V E F$ right ventricle ejection fraction, $\mathrm{SUV}$ standardized uptake value, $\mathrm{SVO}_{2}$ mixed venous oxygen saturation, WHO World Health Organisation

* Remained statistically significant in multivariable stepwise regression model included $\mathrm{PAH}$ prognostic parameters (WHO class, $\mathrm{SUV}_{\mathrm{RV}} / \mathrm{SUV}_{\mathrm{LV}}, \mathrm{RVEF}, \mathrm{mPAP}$ ), $\left(R^{2}=0.68, p<0.001\right)$

or CMR) was used as a surrogate of RV contractility and echo RV systolic pressure as a surrogate of RV afterload. There were significant differences in TAPSE/RVSP values between $\mathrm{CEP}(+)$ and $\mathrm{CEP}(-)$ groups. Similar results we also got with other non-invasive coupling parameters-RV GLS/RVSP (CMR/echo); TAPSE/RVSP (CMR/echo) and RVEF/RVSP (CMR/echo), Table 3. However, all non-invasive coupling parameters had lower AUCs in predicting CEP than RV GLS/PASP, Table 4.

\section{Discussion}

This is the first study to our knowledge which: (1) confirms prognostic value of indexed CMR-derived RV GLS by $\mathrm{RHC}$-derived variables describing afterload in $\mathrm{PAH}$ patients and (2) combines RV-arterial coupling parameters with metabolic alterations of RV myocytes using multimodal approach.

$\mathrm{PAH}$ is a condition associated with high mortality, even in young patients. The prognosis is strictly related to RV function, one of the main predictors of longterm outcome in patients with pulmonary hypertension irrespective of its etiology [20]. Our study population consisted of stable patients with mostly idiopathic, moderate-to-severe PAH (basing on PAP). Mean standard, hemodynamic, prognostic parameters (according to ESC guidelines) e.g. mPAP or cardiac index were overall unfavorable; whereas mean 6MWT distance or BNP levels were mostly in normal ranges or barely deteriorated. These suggest need for more accurate assessment and better prognostication of $\mathrm{PAH}$ patients with already moderate risk of death.

In PAH, RV load could increase even fivefold, thus coupling can only be achieved by an almost similar increase in contractility [6]. We hypothesized that quantification of RV-arterial coupling based on RV GLS as a contractility parameter may offer additive value. It was confirmed that RV strain mirrors RV-arterial uncoupling and its assessment provides information on the adaptation of RV inotropic function to afterload [13].

To obtain RV GLS, CMR feature tracking analysis was used. This method has been validated against myocardial tagging for LV strain analysis and recently against RV speckle tracking imaging for RV longitudinal strain evaluation in tetralogy of Fallot patients or arrhythmogenic RV dysplasia [14]. Systematic evaluation of RV strain in $\mathrm{PH}$ with CMR feature tracking seems to be feasible, reproducible and it was proven that reduced RV strain is associated with subsequent clinical deterioration. Our findings are also consistent with those reported before with the use of echocardiography-patients with worse prognosis had less negative RV GLS [21]. Haeck et al. evaluated 150 patients with $\mathrm{PH}$ and demonstrated that echo-derived RV GLS is a significant determinant of all-cause mortality risk compared to patients with higher GLS (threshold of $<-19 \%$ ) [12]. In our study the cut-off value was $<-16 \%$. We are aware, that RV GLS represents only longitudinal shortening and when PAH advances, RV becomes also more hypertrophic and circumferential deformation becomes also important $[12,13]$. However, RV GLS in our study strongly correlated with other RV systolic function parameters e.g. TAPSE or FAC. Research by di Siqueira et all proved that CMR derived RV GLS was independently associated with the CEP after adjustment for a few clinically meaningful covariates e.g. cardiac index [14].

In this study, we propose indexation of RV GLS with RHC-derived surrogates of RV afterload (PASP; PVR; 
PAC) as that may provide even more accurate estimation of RV-arterial coupling in PAH [4]. The RV is a pulsatile pump and its efficiency depends on sustained hemodynamic coupling with the compliant pulmonary circulation. The resistance and compliance of the pulmonary vasculature contribute to the RV afterload, which comprises the steady and unsteady loads that oppose the ejection of blood during ventricular systole. The RV afterload is lower than the LV afterload, both in terms of mean and pulsatile and its rapid changes e.g. during acute pulmonary embolism could be fatal for patient $[15,22]$. There is a growing appreciation of the prognostic value of pulmonary arterial compliance in PAH and some evidence indicates PAC is a better predictor of outcomes than PVR. The most feasible option to obtain PAC is a ratio of stroke volume to pulmonary artery pulse pressure measured by RHC. Despite of the fact that this method overestimates real compliance, hence does not include amount of blood flow from the pulmonary circulation into the capillary bed during systole, it was proven as significant prognostic factor in PAH [23]. Interestingly, some studies confirmed that low PAC (and worse prognosis) was observed in PAH patients, even when PVR was normal. This suggest that PAC could be a cause rather than a consequence of distal small-vessel proliferative vasculopathy [12]. In our study, PAC did not correlate with neither 6MWT distance, BNP, nor RVEF. However, RV GLS*PAC ratio was in strong relationship with hemodynamic parameters, FDG uptake and prognosis. Our results showed that PAC only in combination with contractility parameter have prognostic value in stable PAH patients. This indicates the importance of the holistic approach for RV function assessment e.g. using coupling analysis.

In PAH decreased pulmonary artery compliance and increased pulse wave velocity create reflected waves which appear during mid or late systole, resulting in increased PASP, pulse pressure, and RV pulsatile afterload $[3,12]$. The elevated PASP increases RV wall stress and oxygen consumption. This over time leads to RV hypertrophy and dilation, lower CO (due to RV-pulmonary artery uncoupling) and accompanying higher glucose demand of RV myocytes. In more severe cases areas of fibrosis or inflammation appear in RV insertion points that when assessed by late gadolinium enhancement indicate higher long term risk of adverse outcome [11]. In our study, the highest area under curve in ROC analysis for predicting clinical end-point had RV GLS/ PASP. Although differences between this ratio and other proposed coupling parameters were not significant, only RV GLS/PASP was significantly associated with CEP in multivariable stepwise regression analysis.

$\mathrm{RV}$-arterial coupling may also be presented with a use of other surrogates of RV contractility derived from CMR imaging, such as RVEF or SV divided by end-systolic volume - SV/ESV [24, 25]. Although, RVEF was statistically significant in univariable regression analysis for the CEP, none of RVEF-coupling parameters achieved statistically significant prognostic value. RVEF still remain one the most important prognostic factors in PAH [1], but it may be not the best surrogate in estimating RV-arterial coupling. Recent papers confirmed that increased pulmonary arterial pressures are associated with increases in FDG uptake and that patients with higher $\mathrm{SUV}_{\mathrm{RV} / \mathrm{LV}}$ had significantly worse prognosis $[18,26]$. In our study, PAH patients had higher glucose uptake than control group and $\mathrm{SUV}_{\mathrm{RV} / \mathrm{LV}}$ significantly correlated with various hemodynamic parameters. Furthermore, in the current study we managed to confirm that worse values of coupling parameters are linked with altered glucose metabolism in PAH patients' heart. This is in agreement with previous research, which suggested that if Ees/Ea ratio and its surrogates are out of normal ranges, decreased mechanical efficiency and loss of ventriculoarterial coupling is observed what requires the increased oxygen consumption [27]. This results in mitochondrial dysfunction and altered myocytes' metabolism. Interestingly, among many parameters only RV GLS/PASP remained statistically associated with $\mathrm{SUV}_{\mathrm{RV} / \mathrm{LV}}$ dichotomized at 1 what suggests a strong relationship between declining RV-arterial coupling and altered RV myocytes' metabolism. Both phenomena preceded clinical deterioration in hemodynamically stable PAH patients.

We are aware that glucose uptake measured using one tracer (FDG) in PET imaging does not fully reflects real "metabolic shift" of RV myocytes into glycolysis but $\mathrm{SUV}_{\mathrm{RV} / \mathrm{LV}}$ ratio parameter is now considered as an established surrogate in many papers $[17,18,27]$. Therefore, we would like to underline this possible connection between coupling parameters and glucose uptake. Subtle changes both in cardiac metabolism and RV-arterial coupling seem to be an interesting foundation for new research in this field.

\section{Limitations}

The main limitations of this study are relatively small patient population and that only initial measurements at baseline visit were analysed. However, study group size is equivalent to similar previous surveys in this field. Coupling parameters, e.g. RV GLS/PASP, RV GLS/PVR and RV GLS*PAC ratios are simplified estimations and we did not use direct measurements from pressure-volume loops, but many previous studies used similar formulas and confirmed their significance in PAH patient evaluation [28]. Another limitation is that PET/CMR images were obtained in patients in stable condition, so the intrinsic diversity of the group is 
limited in comparison to the unselected patients seen in everyday practice.

\section{Conclusions}

Multimodal approach (PET/CMR hybrid and heart catheterization) allows to confirm the relationship between RV-arterial uncoupling and higher metabolic demand that heralds the clinical deterioration of PAH patients. Coupling parameters like RV GLS/PASP may add additional prognostic value in PAH patients' assessments but this requires further larger studies.

\begin{abstract}
Abbreviations
6MWT: Six minute walk test; BNP: Brain natriuretic peptide; BSA: Body surface area; bSSFP: Balanced steady state free precession; CEP: Composite end-point; CMR: Cardiovascular magnetic resonance; CO: Cardiac output; dPAP: Diastolic pulmonary artery pressure; Ea: Arterial elastance; Ees: End-systolic elastance; ESC: European Society of Cardiology; FAC: Fractional area change; FDG: ${ }^{18}$ F-fluorodeoxyglucose; GLS: Global longitudinal strain; LV: Left ventricle/left ventricular; LVEF: Left ventricular ejection fraction; mPAP: Mean pulmonary artery pressure; PAC: Pulmonary artery compliance; PAH: Pulmonary arterial hypertension; PASP: Pulmonary artery systolic pressure; PAWP: Pulmonary artery wedge pressure; PET: Positron emission tomography; PH: Pulmonary hypertension; PVR: Pulmonary vascular resistance; RHC: Right heart catheterization; ROC: Receiver operator characteristic; RV: Right ventricle/right ventricular; RVEF: Right ventricle ejection fraction; SPAP: Systolic pulmonary artery pressure; SUV: Standardized uptake value; SV: Stroke volume; TAPSE: Tricuspid annular plane systolic excursion; WHO: World Health Organization; WU: Wood's Units.
\end{abstract}

\section{Acknowledgements}

Authors would like to thank Jacek Jamiolkowski MD MSc PhD for statistical analysis and invaluable advice.

\section{Authors' contributions}

RK: took part in all activities related to the conduct of the study, data collection, statistical analysis, discussion and writing of the manuscript. LAM: analysis of CMR results, discussion and writing of the manuscript. PS, SGN: PET results analysis. PB: contributed to the data collection, editing of the manuscript. DJ: contributed to the data collection. MH: preparation of PET/CMR procedures and scans. BS, JM, RG, WJM: contributed to data collection and the editing of the manuscript. KAK: oversaw all activities related to the conduct of the study and devised the study idea, contributed to the statistical analysis, discussion writing and editing of the manuscript. All authors read and approved the final manuscript.

\section{Funding}

This work was supported by National Center for Science in Poland ["Preludium" Grant 2017/25/N/NZ5/02689 to R.K.], statutory grant of Medical University of Bialystok and by Leading National Research Center in Bialystok.

\section{Availability of data and materials}

The datasets generated and/or analyzed during the current study are available from the corresponding author on reasonable request.

\section{Declarations}

Ethics approval and consent to participate

The study protocol was reviewed and approved by each institutions' review board that waived the need for specific informed consent.

\section{Consent for publication}

Not applicable.

\section{Competing interests}

The authors declare that they have no competing interests.

\section{Author details}

${ }^{1}$ Department of Cardiology, Medical University of Bialystok, Białystok, Poland. ${ }^{2}$ Department of Epidemiology, Cardiovascular Disease Prevention and Health Promotion, National Institute of Cardiology, Warsaw, Poland. ${ }^{3}$ Laboratory of Molecular Imaging, Medical University of Bialystok, Białystok, Poland. ${ }^{4}$ Department of Nuclear Medicine, Medical University of Bialystok, Białystok, Poland. ${ }^{5}$ Department of Nuclear Medicine, Technical University Munich, Munich, Germany. ${ }^{6}$ Department of Cardiology, Cardinal Wyszynski' Hospital, Lublin, Poland. ${ }^{7}$ Department of Population Medicine and Lifestyle Diseases Prevention, Medical University of Bialystok, Waszyngtona 13a, Białystok 15-269, Poland.

Received: 13 June 2020 Accepted: 16 March 2021

Published online: 10 May 2021

\section{References}

1. Galiè N, Humbert M, Vachiery JL, et al. ESC Scientific Document Group. Guidelines for the diagnosis and treatment of pulmonary hypertension: the task force for the diagnosis and treatment of pulmonary hypertension of the European Society of Cardiology (ESC) and the European Respiratory Society (ERS), endorsed by the International Society of Heart and Lung Transplantation (ISHLT). Eur Heart J. 2016;37:67-119.

2. Champion HC, Michelakis ED, Hassoun PM. Comprehensive invasive and noninvasive approach to the right ventricle pulmonary circulation unit: state of the art and clinical and research implications. Circulation. 2009:120:992-1007.

3. Seo HS, Lee H. Assessment of right ventricular function in pulmonary hypertension with multimodality imaging. J Cardiovasc Imaging. 2018;26(4):189-200

4. Vonk Noordegraaf A, Westerhof BE, Westerhof $\mathrm{N}$. The relationship between the right ventricle and its load in pulmonary hypertension. J Am Coll Cardiol. 2017;69(2):236-43.

5. Bellofiore A, Chesler NC. Methods for measuring right ventricular function and hemodynamic coupling with the pulmonary vasculature. Ann Biomed Eng. 2013:41(7):1384-98.

6. Ryan J, Huston J, Kutty S, et al. Right ventricular adaptation and failure in pulmonary arterial hypertension. Can J Cardiol. 2015;31(4):391-406.

7. Benza RL, Biederman R, Murali S, Gupta H. Role of cardiac magnetic resonance imaging in the management of patients with pulmonary arterial hypertension. J Am Coll Cardiol. 2008;52:1683-92.

8. McLure LE, Peacock AJ. Cardiac magnetic resonance imaging for the assessment of the heart and pulmonary circulation in pulmonary hypertension. Eur Respir J. 2009;33:1454-66.

9. Bradlow WM, Hughes ML, Keenan NG, et al. Measuring the heart in pulmonary arterial hypertension (PAH): implications for trial study size. J Magn Reson Imaging. 2010;31:117-24.

10. Hardegree EL, Sachdev A, Villarraga HR, et al. Role of serial quantitative assessment of right ventricular function by strain in pulmonary arterial hypertension. Am J Cardiol. 2013;111(1):143-8.

11. Kazimierczyk R, Malek LA, Szumowski P, Blaszczak P, Jurgilewicz D, Hladunski $M$, et al. Prognostic value of late gadolinium enhancement mass index in patients with pulmonary arterial hypertension. Adv Med Sci. 2020. https://doi.org/10.1016/j.advms.2020.11.002.

12. Haeck ML, Scherptong RW, Marsan NA, et al. Prognostic value of right ventricular longitudinal peak systolic strain in patients with pulmonary hypertension. Circ Cardiovasc Imaging. 2012;5(5):628-36.

13. lacoviello M, Monitillo F, Citarelli G, et al. Right ventriculo-arterial coupling assessed by two-dimensional strain: a new parameter of right ventricular function independently associated with prognosis in chronic heart failure patients. Int J Cardiol. 2017;241:318-21.

14. de Siqueira ME, Pozo E, Fernandes VR, et al. Characterization and clinical significance of right ventricular mechanics in pulmonary hypertension evaluated with cardiovascular magnetic resonance feature tracking. J Cardiovasc Magn Reson. 2016;18(1):39. 
15. Thenappan T, Prins KW, Pritzker MR, Scandurra J, Volmers K, Weir EK. The critical role of pulmonary arterial compliance in pulmonary hypertension. Ann Am Thorac Soc. 2016;13(2):276-84.

16. Ohira $H$, deKemp $R$, Pena $E$, et al. Shifts in myocardial fatty acid and glucose metabolism in pulmonary arterial hypertension: a potential mechanism for a maladaptive right ventricular response. Eur Heart J Cardiovasc Imaging. 2016;17(12):1424-31.

17. Li W, Wang $\mathrm{L}$, Xiong CM, et al. The prognostic value of $18 \mathrm{~F}-\mathrm{FDG}$ uptake ratio between the right and left ventricles in idiopathic pulmonary arterial hypertension. Clin Nucl Med. 2015;40(11):859-63.

18. Kazimierczyk R, Szumowski P, Nekolla SG, Blaszczak P, Malek L, MiloszWieczorek B, et al. Prognostic role of PET/MRI hybrid imaging in patients with pulmonary arterial hypertension. Heart. 2020. https://doi.org/10. 1136/heartjnl-2020-316741.

19. Szychta W, Werys K, Barczuk-Falęcka M, Postula M, Malek LA. Validation of performance of free of charge plugin for the open-source platform to perform cardiac segmentation in magnetic resonance imaging. Heart Beat. 2018;3:83-9.

20. Benza RL, Miller DP, Gomberg-Maitland M, et al. Predicting survival in pulmonary arterial hypertension: insights from the Registry to Evaluate Early and Long-Term Pulmonary Arterial Hypertension Disease Management (REVEAL). Circulation. 2010;122:164-72.

21. Park JH, Park MM, Farha S, et al. Impaired global right ventricular longitudinal strain predicts long-term adverse outcomes in patients with pulmonary arterial hypertension. J Cardiovasc Ultrasound. 2015;23(2):91-9.

22. Vonk-Noordegraaf A, Haddad F, Chin KM, et al. Right heart adaptation to pulmonary arterial hypertension: physiology and pathobiology. J Am Coll Cardiol. 2013;62:D22-33
23. Al-Naamani N, Preston IR, Hill NS, Roberts KE. The prognostic significance of pulmonary arterial capacitance in pulmonary arterial hypertension: single-center experience. Pulm Circ. 2016;6(4):608-10.

24. Vanderpool RR, Rischard F, Naeije R, Hunter K, Simon MA. Simple functional imaging of the right ventricle in pulmonary hypertension: can right ventricular ejection fraction be improved? Int J Cardiol. 2016;223:93-4.

25. Vanderpool RR, Pinsky MR, Naeije R, et al. RV-pulmonary arterial coupling predicts outcome in patients referred for pulmonary hypertension. Heart. 2015;101:37-43.

26. Yang $T$, Wang $L$, Xiong $C M$, et al. The ratio of (18)F-FDG activity uptake between the right and left ventricle in patients with pulmonary hypertension correlates with the right ventricular function. Clin Nucl Med. 2014;39(5):426-30.

27. Tello K, Dalmer A, Vanderpool R, et al. Cardiac magnetic resonance imaging-based right ventricular strain analysis for assessment of coupling and diastolic function in pulmonary hypertension. JACC CardiovasC Imaging. 2019;12(11 Pt 1):2155-64.

28. Tello K, Wan J, Dalmer A, et al. Validation of the tricuspid annular plane systolic excursion/systolic pulmonary artery pressure ratio for the assessment of right ventricular-arterial coupling in severe pulmonary hypertension. Circ Cardiovasc Imaging. 2019;12(9):e009047.

\section{Publisher's Note}

Springer Nature remains neutral with regard to jurisdictional claims in published maps and institutional affiliations.
Ready to submit your research? Choose BMC and benefit from:

- fast, convenient online submission

- thorough peer review by experienced researchers in your field

- rapid publication on acceptance

- support for research data, including large and complex data types

- gold Open Access which fosters wider collaboration and increased citations

- maximum visibility for your research: over 100M website views per year

At BMC, research is always in progress.

Learn more biomedcentral.com/submissions 